\title{
Takayasu Arteritis with Cerebral Hypoperfusion: An Unusual Case
}

Sir,

Takayasu arteritis (TA) is a chronic inflammatory disease mainly involving aorta and its major branches. We report the anesthetic management of a 12-year-old male child suffering from TA-induced chronic cerebral hypoxemia for which he was operated for aortocarotid bypass using polytetrafluoroethylene (PTFE) graft under general anesthesia. Postoperatively, the patient developed airway edema leading to sudden respiratory insufficiency. We discuss nuances of management of such a case with discussion of customized approach of emergency exploration of the neck. The patient presented with weakness of the right upper limb (UL) and easy fatigability. Distal pulses in both ULs were weak in comparison to pulsations in both lower limbs. Noncontrast computed tomography (CT) images showed left-sided infarct in temporoparietal region suggestive of recent stroke. The patient was started on prednisolone and methotrexate. Routine investigations including hemogram, renal and liver function tests and coagulation profile were within normal limits. Carotid Doppler showed circumferential mural thickening involving bilateral common carotid artery, external carotid artery, and internal carotid artery with significant luminal narrowing more on the right side. CT angiography revealed complete occlusion of bilateral subclavian artery, near total occlusion of bilateral common carotid artery, complete occlusion of the right vertebral artery origin, and decreased caliber of the left internal carotid artery with complete occlusion of the left middle cerebral artery. On the basis of clinicoradiologic findings, the diagnosis of TA was established.

The patient was planned for aortic-carotid bypass on the left side. Following anesthetic induction, ultrasound-assisted central venous cannulation using $7 \mathrm{~F}$ catheter in the right subclavian and arterial cannulation in the right dorsalis pedis artery was done as the pulses were not palpable in the ULs. Intraoperatively, the neck was dissected and carotid vessels were exposed on the left side followed by sternotomy. PTFE graft was anastomosed establishing communication made between aorta and left internal carotid artery extending up to the bulb. Postoperatively, the patient was electively ventilated for $24 \mathrm{~h}$ in neurosurgical intensive care unit (ICU) and extubated on the $1^{\text {st }}$ postoperative day (POD). The patient showed signs of upper airway obstruction after extubation. A diffuse swelling was noticed on the left side of the face and neck [Figure 1a]. The airway was immediately secured with 6-mm endotracheal tube using pediatric bougie. Chest X-ray showed unexplained

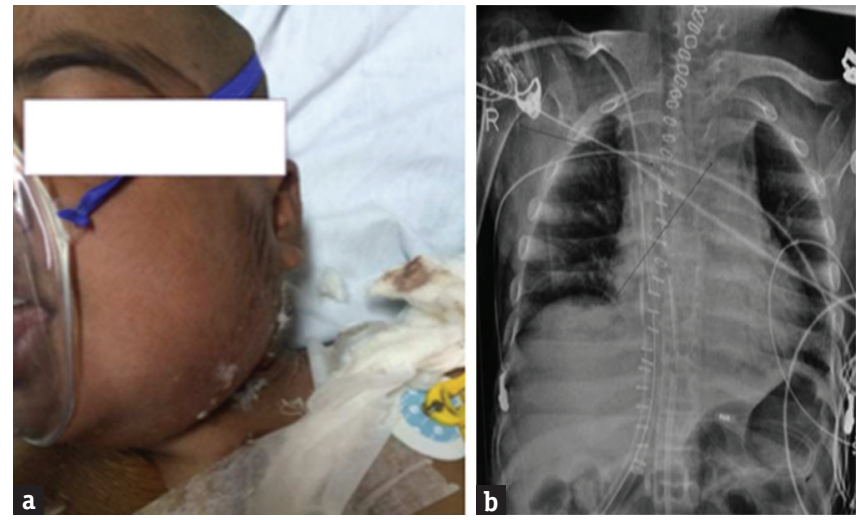

Figure 1: (a) Diffuse swelling on the left side of the face and neck (b) Widened mediastinum on chest X-ray

widening of mediastinum [Figure 1b] suggestive of surgical site collection. The patient was immediately shifted to the emergency operating room for surgical site reexploration. Intraoperatively, no collection could be detected in the neck and chest. The thoracic cavity was closed with intercostal drainage tubes on both sides. There was gross soft-tissue edema in the neck. The patient was reversed and shifted on endotracheal tube back to neurosurgical ICU for elective ventilation. The patient was electively ventilated till swelling subsided and extubated successfully on the $4^{\text {th }}$ POD. The patient was later discharged without any neurological deficits.

Takayasu disease is a multisystem connective tissue disorder and has varied presentation, which probably causes delayed diagnosis and treatment. Patients usually present with vague symptoms such as fever, malaise, fatigue, and arthralgia. Hypertension, arterial bruit, and asymmetrical presence of distal pulses are important clue in the diagnosis. Early initiation of immunosuppressant's such as steroids or methotrexate remains mainstay of therapy. ${ }^{[1-3]}$ In cases of major stenotic and occlusive lesions, revascularization may be needed. Various early postoperative complications have been described in the literature such as cerebral hyperperfusion syndrome with intracerebral hemorrhage, cardiac tamponade, and arterial dissection $^{[4]}$ after successful PTFE graft. In the present case, the probable cause for swelling was either venous edema or lymphatic edema. Venous and soft-tissue edema is attributable to excessive handling of the tissues following prolonged surgery. Lymphatic edema tends to appear few days after surgery and not in the immediate postoperative period. As a surgical dictum, neck swelling sufficient enough to cause respiratory insufficiency after any surgery in the neck warrants 
exploration to rule out hematoma in the operative field. Such complications have been described in detail in the literature, especially after thyroidectomy, carotid endarterectomy, and anterior cervical spine surgeries. Very rarely, respiratory discomfort may arise due to subcutaneous emphysema and soft-tissue edema. In the present case, local edema at operative site causing airway obstruction and mediastinal widening on chest X-ray, mimicked focal collection (suggestive of hematoma) and led to unnecessary emergency reexploration. In such a situation, securing a patent airway should follow principles of resuscitation. Once adequately secured, the patient should be immediately evaluated with CT scan neck. CT scan is a time efficient investigation, which can be performed in a 5-min period and confidently rules out any fluid collection, especially blood. A negative finding can be later confirmed with ancillary investigation such as magnetic resonance imaging once the patient is stabilized.

\section{Declaration of patient consent}

The authors certify that they have obtained all appropriate patient consent forms. In the form, the legal guardian has given his consent for images and other clinical information to be reported in the journal. The guardian understands that names and initials will not be published and due efforts will be made to conceal patient identity, but anonymity cannot be guaranteed.

\section{Financial support and sponsorship}

Nil.

\section{Conflicts of interest}

There are no conflicts of interest.
Rajeev Chauhan, Ankur Luthra, Sachin Mahajan ${ }^{1}$

Departments of Anaesthesia and Intensive Care and ${ }^{1}$ CTVS, PGIMER, Chandigarh, India

Address for correspondence: Dr. Ankur Luthra, Department of Anaesthesia and Intensive Care, PGIMER,

Chandigarh, India.

E-mail: zazzydude979@gmail.com

\section{REFERENCES}

1. Mason JC. Takayasu arteritis - Advances in diagnosis and management. Nat Rev Rheumatol 2010;6:406-15.

2. Koide K. Takayasu arteritis in Japan. Heart Vessels Suppl 1992;7:48-54.

3. Waern AU, Andersson P, Hemmingsson A. Takayasu's arteritis: A hospital-region based study on occurrence, treatment and prognosis. Angiology 1983;34:311-20.

4. Kim YW, Kim DI, Park YJ, Yang SS, Lee GY, Kim DK, et al. Surgical bypass vs. endovascular treatment for patients with supra-aortic arterial occlusive disease due to takayasu arteritis. J Vasc Surg 2012;55:693-700.

This is an open access journal, and articles are distributed under the terms of the Creative Commons Attribution-NonCommercial-ShareAlike 4.0 License, which allows others to remix, tweak, and build upon the work non-commercially, as long as appropriate credit is given and the new creations are licensed under the identical terms.

\begin{tabular}{|l|l|}
\hline \multicolumn{2}{c|}{ Access this article online } \\
\hline Quick Response Code: & Website: \\
\hline
\end{tabular}

How to cite this article: Chauhan R, Luthra A, Mahajan S. Takayasu arteritis with cerebral hypoperfusion: An unusual case. J Neurosci Rural Pract 2019;10:170-1.

(c) 2018 Journal of Neurosciences in Rural Practice | Published by Wolters Kluwer - Medknow 